\title{
Integrated Atmosphere Resource Recovery and Environmental Monitoring Technology Demonstration for Deep Space Exploration
}

Jay Perry, Morgan Abney, Jim Knox, Keith Parrish, and Monsi Roman NASA Marshall Space Flight Center

Darrell Jan

NASA Jet Propulsion Laboratory

42nd ICES, 16-19 July 2012, San Diego, California, USA 
Technological Advancement Objectives

- To evolve the ISS environmental control and life support (ECLS) system platform to enable deep space exploration

- Improve reliability \& maintainability

- Reduce consumable mass

- To maximize commonality across missions and vehicles

- To mature process technologies for flight programs

- Reduce technical risk and cost

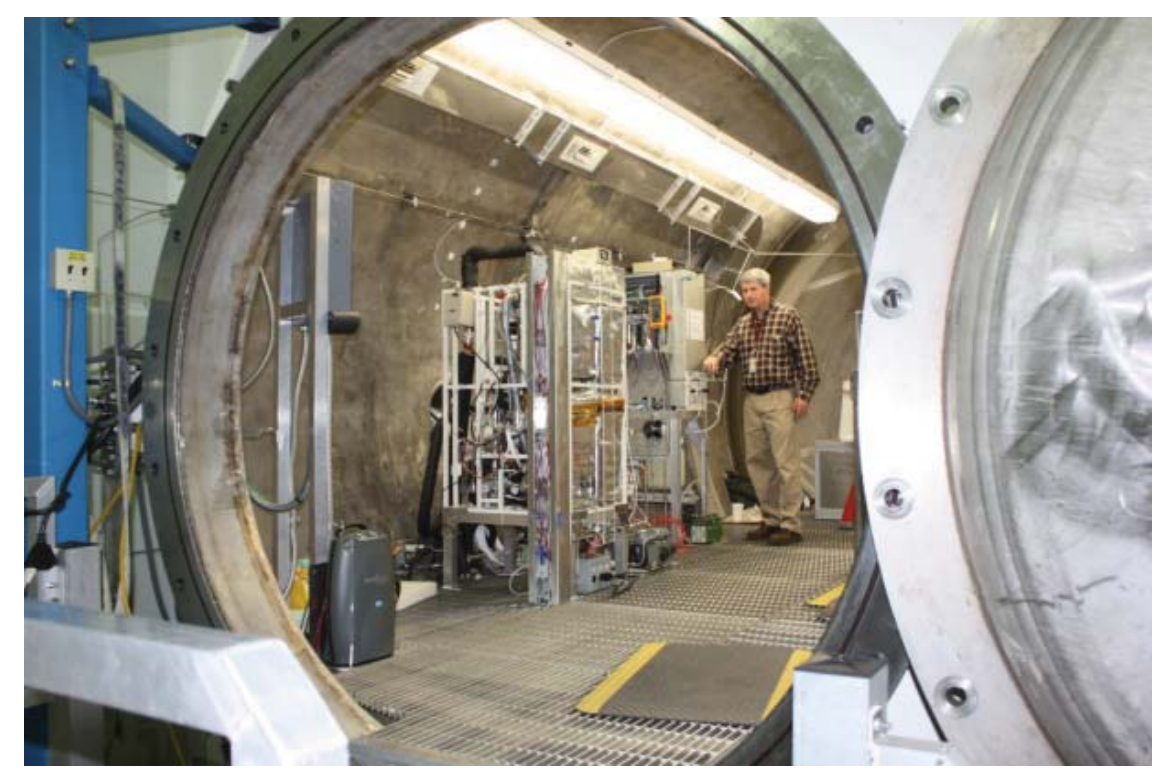

- To develop modular resource recovery technologies 


\section{OAIAA}

\section{What is Atmosphere Revitalization?}

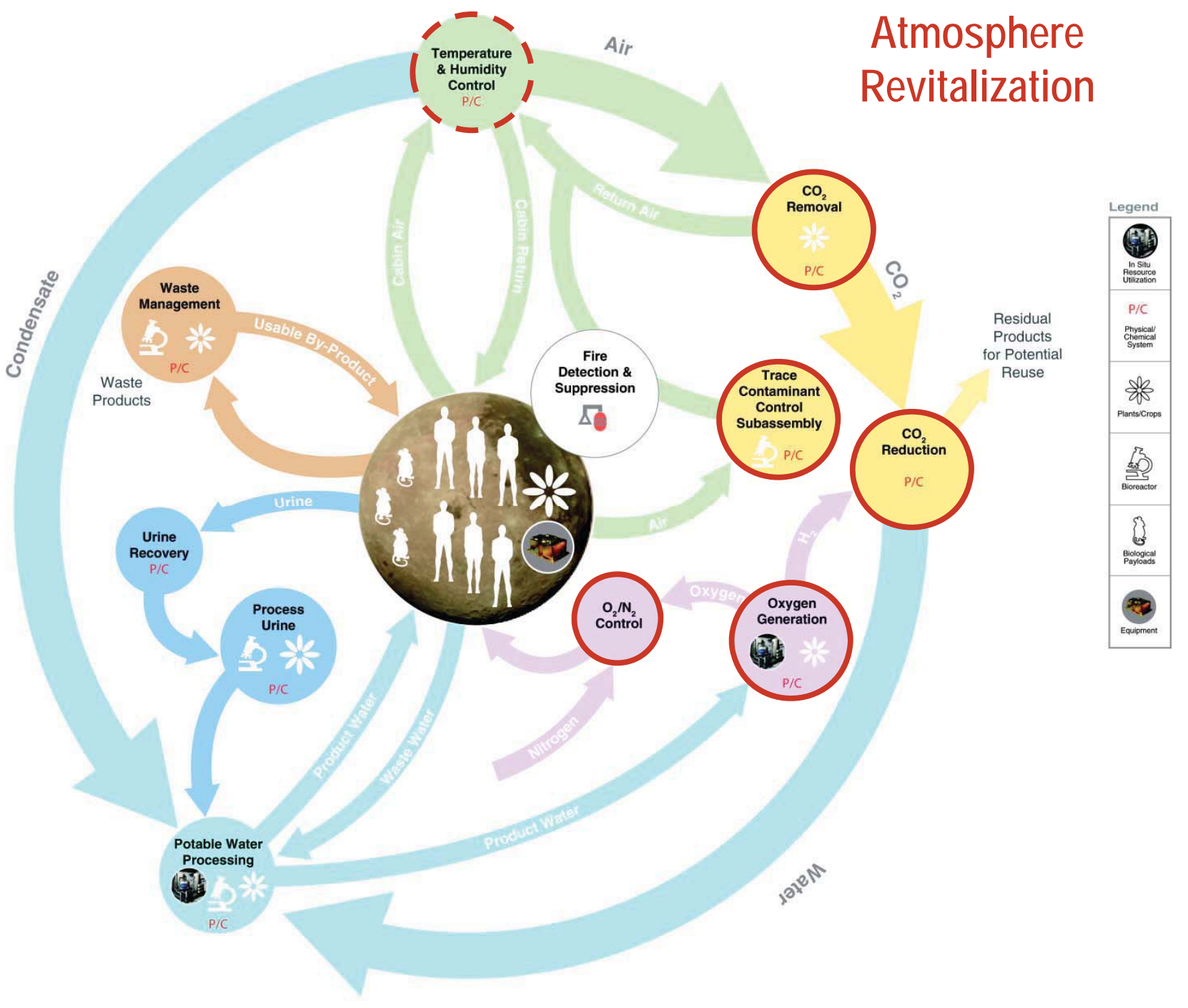




\section{Functional Trade Spaces Help Focus Development}

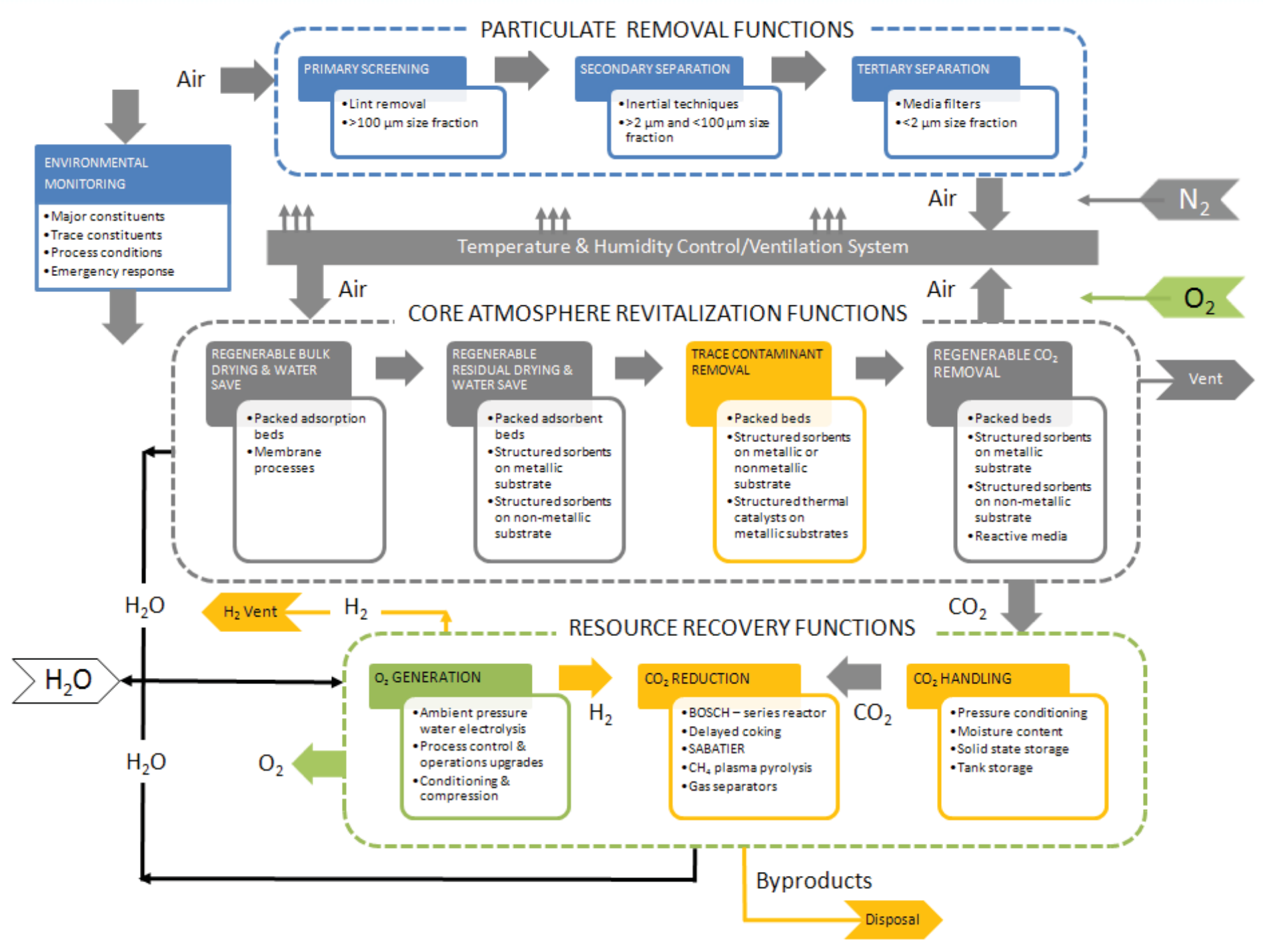




\section{Spacecraft Atmosphere Revitalization Past \& Present}

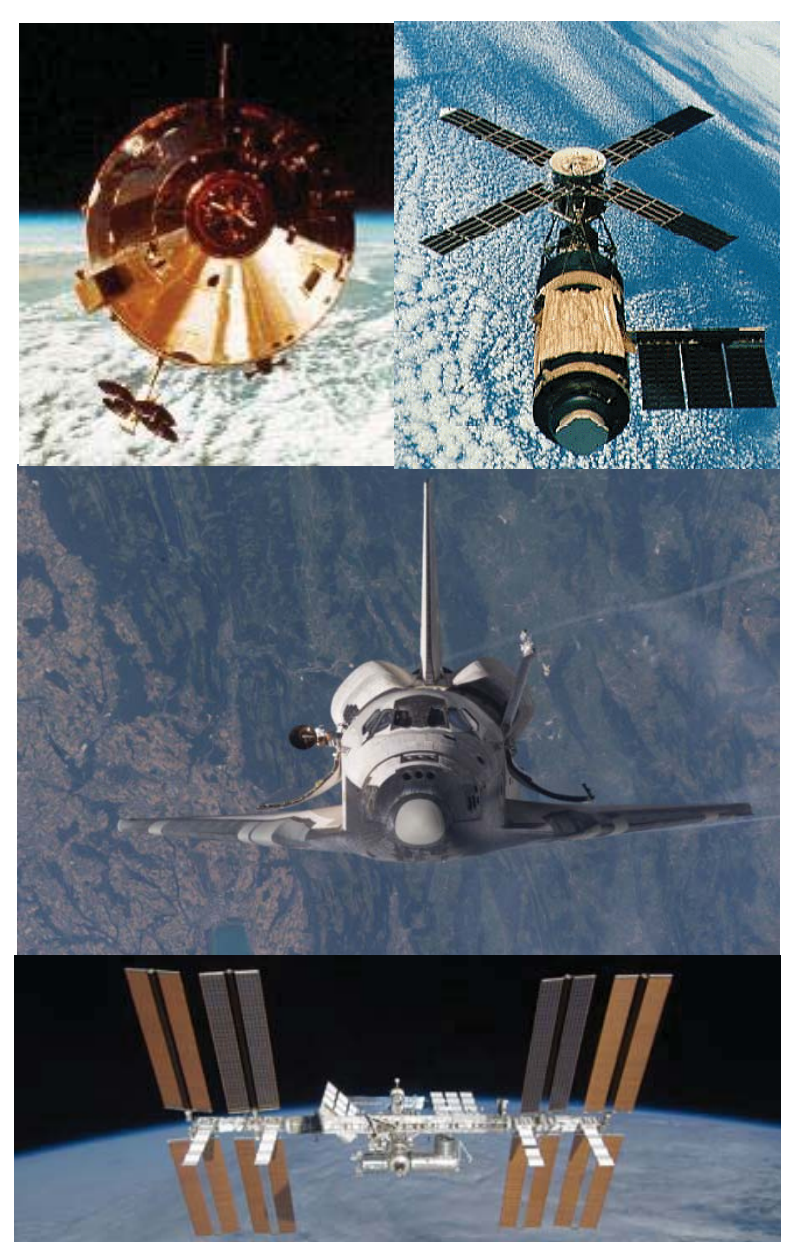

\begin{tabular}{|c|c|c|c|c|}
\hline PROJECT & $\begin{array}{l}\text { MISSION } \\
\text { DURATION }\end{array}$ & $\begin{array}{c}\text { CABIN } \\
\text { VOLUME } \\
\left(\mathbf{m}^{3}\right)\end{array}$ & $\begin{array}{l}\text { CREW } \\
\text { SIZE }\end{array}$ & TECHNOLOGICAL APPROACH \\
\hline Mercury & 34 hours & 1.56 & 1 & $\begin{array}{l}\text { Atmosphere: } 100 \% \mathrm{O}_{2} \text { at } 34.5 \mathrm{kPa} \text {. } \\
\text { Atmosphere supply: Gas at } 51.7 \mathrm{MPa} . \\
\mathrm{CO}_{2} \text { removal: } \mathrm{LiOH} \text {. } \\
\text { Trace contaminants: Activated carbon. }\end{array}$ \\
\hline Gemini & 14 days & 2.26 & 2 & $\begin{array}{l}\text { Atmosphere: } 100 \% \mathrm{O}_{2} \text { at } 34.5 \mathrm{kPa} \text {. } \\
\text { Atmosphere supply: Supercritical storage at } \\
5.86 \mathrm{MPa} \text {. } \\
\mathrm{CO}_{2} \text { removal: } \mathrm{LiOH} \text {. } \\
\text { Trace contaminants: Activated carbon. }\end{array}$ \\
\hline Apollo & 14 days & 5.9 & 3 & $\begin{array}{l}\text { Atmosphere: } 100 \% \mathrm{O}_{2} \text { at } 34.5 \mathrm{kPa} \text {. } \\
\text { Atmosphere supply: Supercritical storage at } \\
6.2 \mathrm{MPa} \text {. } \\
\mathrm{CO}_{2} \text { removal: LiOH. } \\
\text { Trace contaminants: Activated carbon. }\end{array}$ \\
\hline Skylab & 84 days & 361 & 3 & $\begin{array}{l}\text { Atmosphere: } 72 \% \mathrm{O}_{2} / 28 \% \mathrm{~N}_{2} \text { at } 34.5 \mathrm{kPa} . \\
\text { Atmosphere supply: } \mathrm{Gas} \text { at } 20.7 \mathrm{MPa} \text {. } \\
\mathbf{C O}_{2} \text { removal: Type } 13 \mathrm{X} \text { and } 5 \mathrm{~A} \text { molecular } \\
\text { sieves regenerated by vacuum swing. } \\
\text { Trace contaminants: Activated carbon. }\end{array}$ \\
\hline Space Shuttle & 14 days & 74 & 7 & $\begin{array}{l}\text { Atmosphere: } 21.7 \% \mathrm{O}_{2} / 78.3 \% \mathrm{~N}_{2} \text { at } 101 \mathrm{kPa} \\
\text { Atmosphere supply: } \mathrm{Gas} \text { at } 22.8 \mathrm{MPa} \\
\mathrm{CO}_{2} \text { removal: } \mathrm{LiOH} \\
\text { Trace contaminants: Activated carbon and } \\
\text { ambient temperature } \mathrm{CO} \text { oxidation }\end{array}$ \\
\hline $\begin{array}{l}\text { International } \\
\text { Space Station }\end{array}$ & 180 days & Up to 600 & 3 to 6 & $\begin{array}{l}\text { Atmosphere: } 21.7 \% \mathrm{O}_{2} / 78.3 \% \mathrm{~N}_{2} \text { at } 101 \mathrm{kPa} \\
\text { Atmosphere supply: Gas at } 20.7 \mathrm{MPa} / \text { water } \\
\text { electrolysis } \\
\mathrm{CO}_{2} \text { removal: Silica gel with type } 13 \mathrm{X} \text { and } 5 \mathrm{~A} \\
\text { molecular sieves regenerated by } \\
\text { vacuum/temperature swing } \\
\mathrm{CO}_{2} \text { reduction: Sabatier reactor (scar for } \\
\text { future addition) } \\
\text { Trace contaminants: Activated carbon and } \\
\text { thermal catalytic oxidation }\end{array}$ \\
\hline
\end{tabular}




\section{OAIAA}

\section{ISS - The "Launch Platform" to Deep Space}

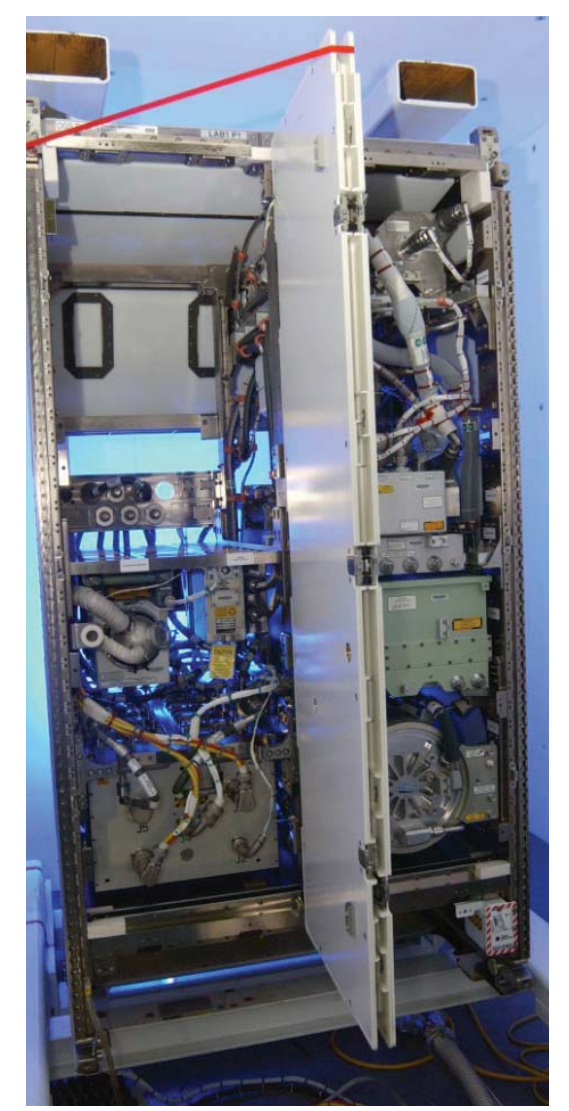

- Reduce:

- Logistics requirements

- Expendable resources

- Complexity

- Improve:

- Operational robustness

- Life cycle economics

- Demonstrate:

- More complete loop closure

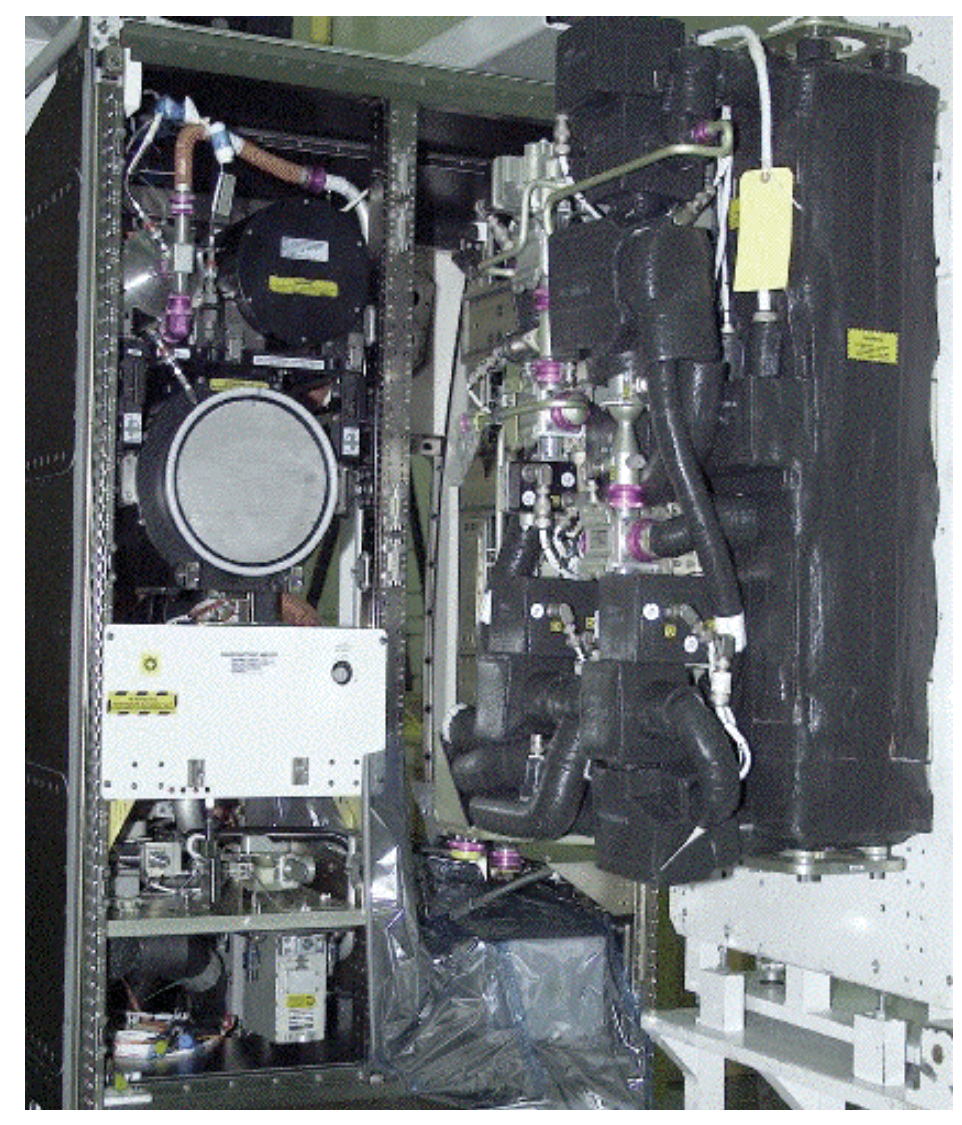




\section{Strategic Improvements}

- Cabin ventilation

- Quiet fan design principles

- Carbon dioxide removal

- Durable adsorbent media

- Process air drying

- Trace contaminant control

- $\quad$ Alternative high capacity adsorbent media

- Structured oxidation catalysts

- Low maintenance particulate filtration \& disposal

- Oxygen supply

- Long-lived electrolysis cell stack materials

- Alternative process control approaches

- Oxygen recovery

- Reduction byproduct processing

- Environmental monitoring

- Alternative major constituent monitoring approaches

- Alternative trace constituent monitoring approaches

- Microbial \& particulate monitoring techniques

\section{OAIAA:}

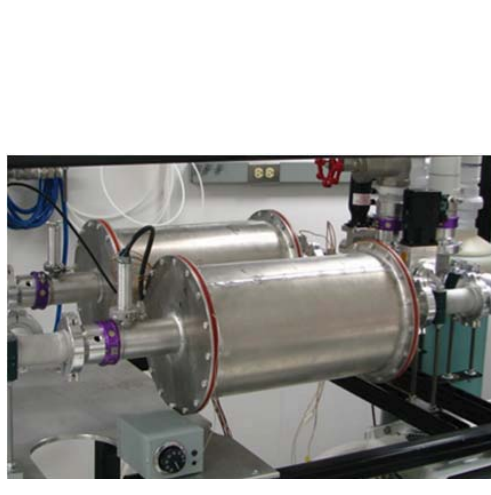

MicrolithAdsorber

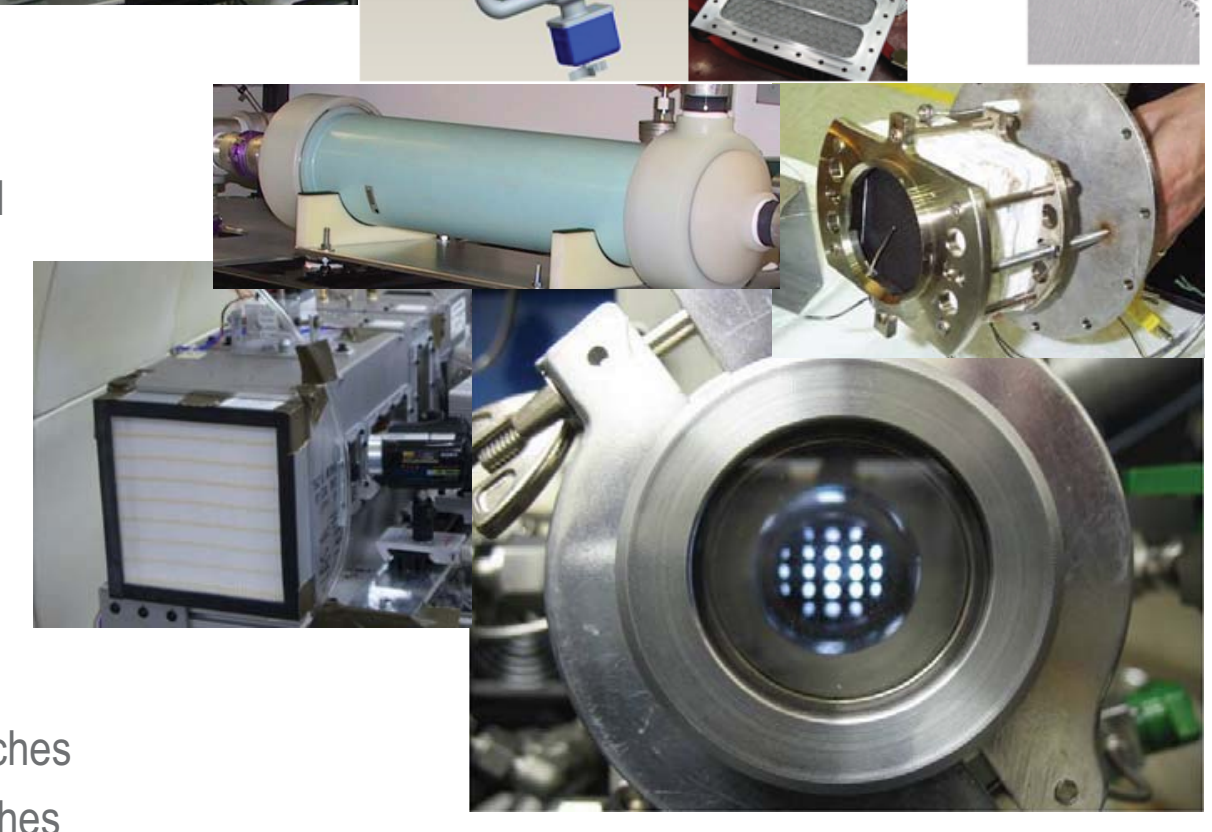




\section{OAIAA:}

\section{ISS Architecture Testing}

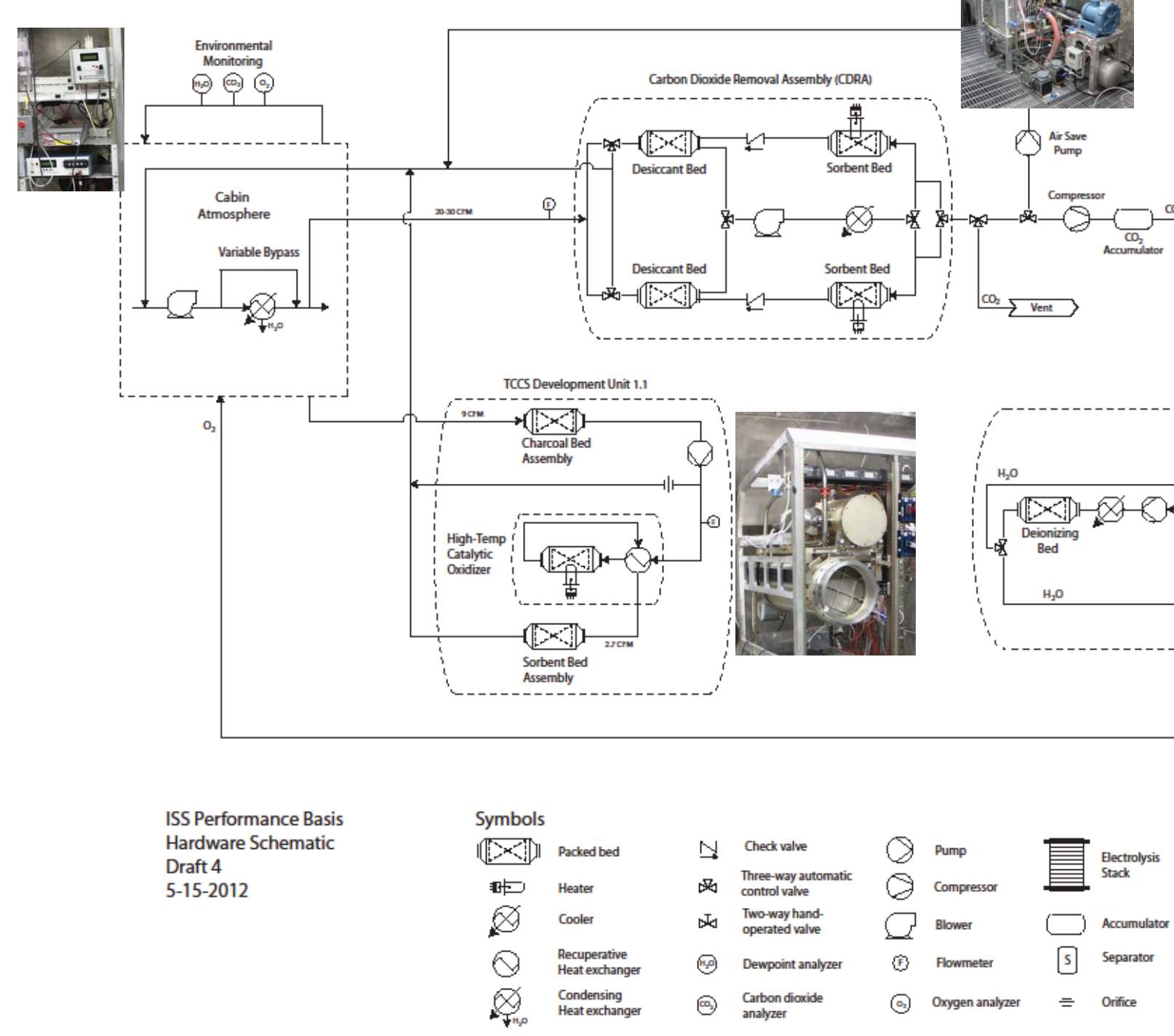




\section{ISS Architecture Testing Objectives}

- Phase 1A-Demonstrate functional performance of the basic ISS AR subsystem using the CDRA in $\mathrm{CO}_{2}$ vent mode and the TCCS operating in parallel.

- Phase 1B-Demonstrate the partial functional performance of the basic ISS AR subsystem when operating in a resource recovery mode that includes integration with $\mathrm{CO}_{2}$ conditioning, storage, and reduction equipment.

- Phase 2 -Investigate propagation of trace contaminants through the core ISS AR subsystem equipment with emphasis on the CDRA and $\mathrm{CO}_{2}$ conditioning and storage equipment.

- Phase 3-Demonstrate the full resource recovery functional performance of the ISS AR subsystem including the $\mathrm{CO}_{2}$ removal, $\mathrm{CO}_{2}$ conditioning and storage, $\mathrm{CO}_{2}$ reduction and post-processing, oxygen generation, and trace contaminant control functions. 


\section{OAIAA:}

\section{Cycle 1 Integrated Process Architecture}

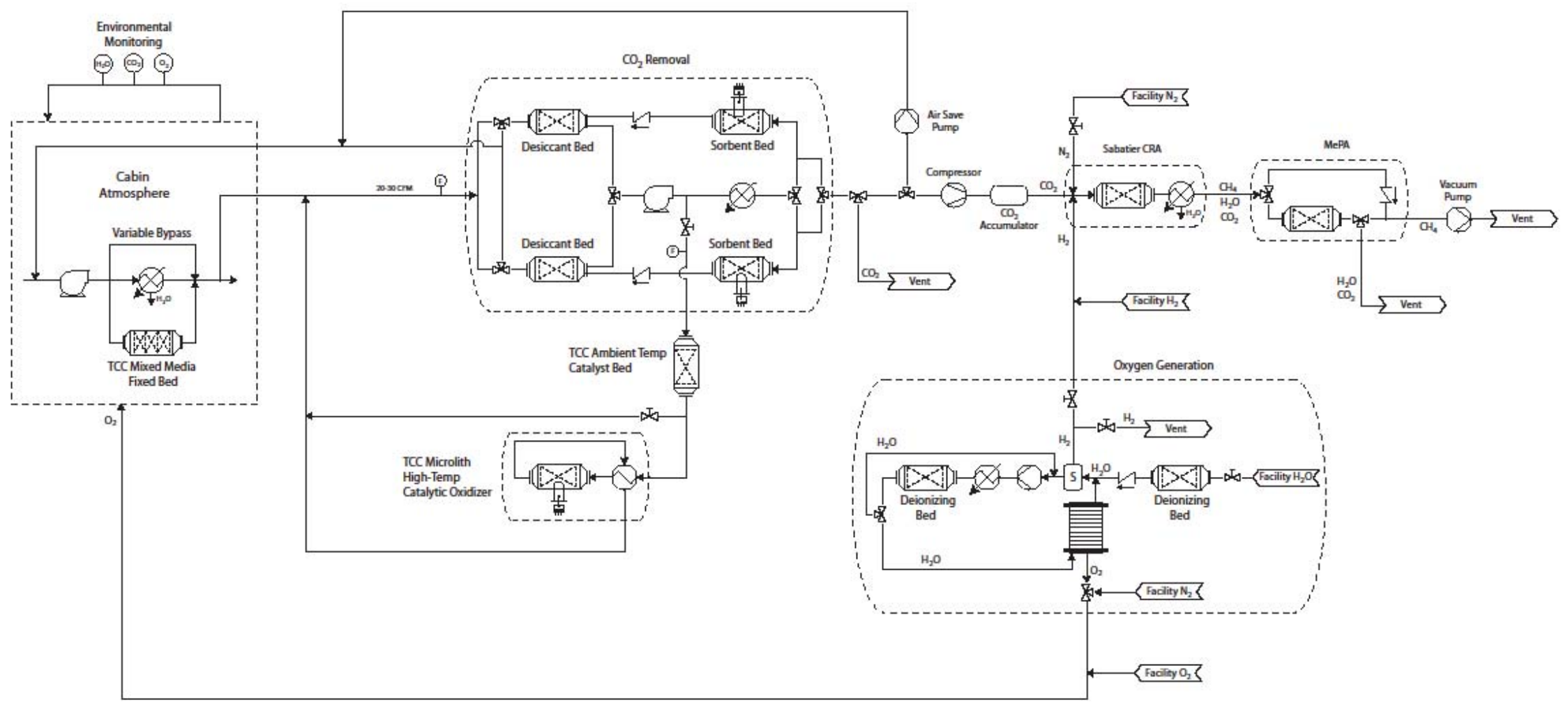

Integrated Systems Test \#1

Symbols

Hardware Schematic

[D. Packed bed

Draft 7

Heater

$\notin$ cooler

(1) Hecuperative

Condensing

N Checkvalve

Three-way automatic
control valve

Qump

㹂 $\begin{gathered}\text { Electrolysis } \\ \text { Stack }\end{gathered}$

Disel Two-way hand-

$Q$ Compressor $\square$ Accumulato

operated value

C. Blower

5 Separator

(9) Carbon diaxide

(1) Howmeter

$\equiv$ Orifice 


\section{Cycle 1 Testing Objectives}

- Demonstrate simultaneous sustained operation of oxygen generation, $\mathrm{CO}_{2}$ removal, trace contaminant control, major constituent monitoring, and $\mathrm{CO}_{2}$ reduction processes under continuous operating conditions using an ISS-derived process architecture.

- Demonstrate the effect of the control algorithm governing the $\mathrm{CO}_{2}$ compressor operation (on/off rules) and the CDRA valve sequencing on the overall $\mathrm{CO}_{2}$ reclamation efficiency for various modes of operation.

- Determine the purity of product $\mathrm{CO}_{2}$ from the CDRA-4 sorbent beds.

- Determine the purity of product oxygen and hydrogen from the OGA.

- Determine the effect cabin atmosphere leakage and/or atmospheric major constituent inclusion on the $\mathrm{CDRA} \mathrm{CO}_{2}$ product may have on CRA performance.

- Determine the purity of product water from the Sabatier-based CRA.

- Demonstrate CRA post-processing first stage to purify methane.

- Demonstrate oxygen generation alternative process control concept. 


\section{Incremental Process Architecture Progression}

- Cycle 1: Modified ISS architecture incorporating improved trace contaminant and $\mathrm{CO}_{2}$ removal adsorbents; trace contaminant removal oxidation catalysts; partial $\mathrm{CO}_{2}$ reduction byproduct processing; and alternative major atmospheric constituent monitoring.

- Cycle 2: Alternative process gas drying equipment; advanced $\mathrm{CO}_{2}$ reduction byproduct processing; and alternative major constituent and volatile organic compound monitoring.

- Cycle 3: Advanced $\mathrm{CO}_{2}$ removal and compression; complete $\mathrm{CO}_{2}$ reduction byproduct processing; advanced environmental monitoring sensor array; ammonia catalytic reduction. 


\section{Conclusion}

- Functional, unit operation-driven approach

- Focus on ISS ECLS system strengths and weaknesses

- Use robust design principles to achieve stage-wise optimization

- Leverage core process technologies from existing equipment designs as appropriate

- Attention to design modularity to address commonality across mission and vehicle architectures 


\section{Further Reading}

- Perry, J.L., Carrasquillo, R.L., and Harris, D.W. (2006) Atmosphere Revitalization Technology Development for Crewed Space Exploration. 44 ${ }^{\text {th }}$ AIAA Aerospace Sciences Meeting and Exhibit. AIAA-2006-140. Reno, Nevada, January 2006.

- Perry, J.L. (2007) Atmosphere Revitalization--Process Technology Maturation for NASA's Constellation Projects. Space Technology and Applications International Forum (STAIF 2007), Albuquerque, New Mexico, February 2007.

- Perry, J.L. and Howard, D.F. (2007) Spacecraft Life Support System Process Technology Maturation using Stage Gate Methodology. $37^{\text {th }}$ International Conference on Environmental Systems. SAE 2007-01-3045.

- Perry, J.L., Bagdigian, R.M., and Carrasquillo, R.L. (2010) Trade Spaces in Crewed Spacecraft Atmosphere Revitalization System Development. AIAA2010-6061, 40 International Conference on Environmental Systems, Barcelona, Spain. 